\title{
ENRIQUECIMIENTO DE HUEVOS CON ÁCIDOS GRASOS OMEGA-3 MEDIANTE LA SUPLEMENTACIÓN CON SEMI LLA DE LINO (Linum usitatissimum) EN LA DIETA
}

\section{EGG ENRI CHMENT WITH OMEGA-3 FATTY ACI DS BY MEANS OF FLAXSEED SUPPLEMENT (Linum usitatissimum) I N THE DIET}

Liliana Betancourt ${ }^{1 *}$, M.Sc, Gonzalo Díaz², Ph.D.

\begin{abstract}
${ }^{1}$ Universidad de La Salle, Facultad de Ciencias Agropecuarias, Laboratorio de Nutrición 2Universidad Nacional de Colombia, Facultad de Medicina Veterinaria y de Zootecnia, Laboratorio de Toxicología y Nutrición, sede Bogotá. *Correspondencia: Icbetancourt@unisalle.edu.co
\end{abstract}

Recibido: Noviembre 25 de 2008; Aceptado: Febrero 25 de 2009

\section{RESUMEN}

Objetivo. Enriquecer los huevos de gallina con ácidos grasos omega-3 (n-3) mediante la inclusión de semillas de lino en la dieta. Materiales y métodos. La dieta de las aves fue formulada con niveles de 0 (L0), 10 (L10), 15 (L15) y 20\% (L20) de linaza. Se determinó la composición de ácidos grasos por cromatografía de gases y se evaluó la producción y calidad del huevo. Resultados. El porcentaje de producción de huevo fue superior en los grupos L0 y L15 ( $p<0.05$ ) con 93,0 y 91,5\% de producción comparada con $86,0 \%$ en los grupos L10 y L20. La mejor conversión de alimento se presentó también en los grupos L0 y L15. Con la inclusión de semilla de lino, se incrementó el contenido de ácidos grasos n-3 desde 3,0 \% en el grupo L0 hasta 12,6\% en el grupo L20, siendo los ácidos grasos n-3 álinolénico (C18:3, n-3) y docosahexahenóico (DHA, C22:6, n-3) los hallados en mayor concentración. Con la inclusión de linaza en la dieta de las gallinas ponedoras se redujo el contenido de acido linoleico ( $18: 2 n-6)$, lo cual resultó en una disminución en la relación n6: n-3. Conclusiones. Con base en la evaluación económica se concluye que es posible enriquecer la yema de huevo de gallina hasta con un 10,1 \% de ácidos grasos n-3 mediante la inclusión de un $15 \%$ de linaza en la dieta de las gallinas, sin embargo, esto representó un sobrecosto de producción del $12 \%$.

Palabras clave: ácido á-linolénico, EPA, DHA, huevo, omega-3. 


\section{ABSTRACT}

Objective. To increase the content of omega- $3(n-3)$ fatty acids in hen's eggs by supplementing flaxseed in the diet. Materials and methods. Four levels of flaxseed amounts were evaluated: $0 \%$ (L0), 10\% (L10), 15\% (L15) and 20\% (L20). We evaluated the fatty acid composition in the egg's yolk, as well as quantity and quality of eggs produced. Results. Percent egg production was higher $(p<0.05)$ in groups L0 $(93.0 \%)$ and L15 $(91.5 \%)$ as compared with groups L10 and L20 (86.0\%). The best feed conversion rate was obtained with treatments L0 and L15. The dietary supplement of flaxseed increased the n- 3 contents in eggs from $3.0 \%$ (in group L0) to $12.6 \%$ (in group L20), being the á-linolenic $(\mathrm{C} 18: 3 \mathrm{n}-3)$ and the docosahexaenoic $(\mathrm{C} 22: 6 \mathrm{n}-3)$ the most abundant fatty acids. Dietary supplement of flaxseed was also responsible for the reduction in the content of linoleic acid (C18:2 n-6), therefore decreasing the $n-6: n-3$ ratio. Conclusions. The economic assessment of the dietary treatments shows that it is feasible to increase the n- 3 fatty acid content of hen's eggs by up to $10.1 \%$ by using $15 \%$ dietary flaxseed; however, this dietary modification represents a $12 \%$ increase in the production costs.

Key words: á-linolenic acid, EPA, DHA, egg, omega-3.

\section{NTRODUCCI ÓN}

La industria de la producción de huevo se ha enfocado tradicionalmente en el mejoramiento de la eficiencia en la producción y el procesamiento; sin embargo, en la actualidad exite preocupación también por el papel de la nutrición animal en el desarrollo de productos diferenciados como los huevos enriquecidos con ácidos grasos omega-3, los cuales pueden tener efectos en la salud humana (1).

La formación de triglicéridos y fosfolípidos en el hígado para la síntesis de yema de huevo, se puede ver afectada por cambios en la composición de la dieta (2). La grasa saturada y monoinsaturada tiene un menor efecto sobre el perfil de ácidos grasos del huevo (3), que la grasa rica en ácidos grasos poliinsaturados, la cual puede causar mayores cambios en el perfil (4), permitiendo de esta manera, la manipulación de la composición de los lípidos de la yema para cubrir los requerimientos nutricionales de los humanos. El interés sobre la relación entre la dieta y la salud humana, proveen oportunidades para la producción y mercado de huevos modificados, por los cuales el consumidor podría pagar un sobrecosto (5).

La inclusión de semilla de lino en la dieta de gallinas ponedoras se ha evaluado en niveles de 10 hasta un 30\% (6), lográndose incrementar en 44 veces el contenido de ácido $\alpha$-linolénico de la yema del huevo, no así, la tasa de deposición de EPA (ácido eicosapentaenoico) y DHA (ácido docosahexaenoico), la cual es baja aun con niveles de inclusión de linaza superiores al $15 \%$ (7). Otro factor que debe considerarse es que la tasa de deposición de ácidos grasos varía con la edad y con la estirpe de las gallinas (8). I gualmente importantes son, los factores antinutricionales que presenta la semilla de lino, como son su baja digestibilidad, contenido de glucósidos cianogénicos y factores antagónicos de la vitamina B6, los cuales restringen el uso de semilla de lino en la dieta de las aves (9).

El objetivo de este trabajo fue, enriquecer los huevos de gallina con ácidos grasos omega-3 (n-3) mediante la inclusión de semillas de lino en la dieta. 


\section{MATERI ALES Y MÉTODOS}

Animales y dietas. Un total de 60 aves de postura de la línea "ISA Brown" de 32 semanas de edad, fueron encasetadas en jaulas y manejadas bajo condiciones comerciales estándar. Se evaluaron cuatro dietas experimentales, incluyendo una dieta control sin inclusión de linaza durante un período de 5 semanas. Las dietas se diseñaron para cubrir los requerimientos de nutrientes para aves de postura (10); los ingredientes y la composición de las dietas se presentan en la tabla 1 . Las dietas se balancearon con niveles de 0 (L0), 10 (L10), 15 (L15) y 20\% (L20) de semilla de lino, todas contenían los mismos niveles de energía y proteína.

Tabla 1. Composición de las diferentes dietas experimentales

\begin{tabular}{|c|c|c|c|c|}
\hline \multirow[b]{2}{*}{ Ingredientes. $\%$} & \multicolumn{4}{|c|}{ Dieta experimental } \\
\hline & L10 & L15 & L20 & Lo \\
\hline Maíz & & & & 48.93 \\
\hline Sorgo & 55.8 & 50.4 & 49.9 & \\
\hline Salvado de trigo & 0.5 & 2.6 & 0.14 & 11.6 \\
\hline Torta de soya $(48 \%)$ & 16.7 & 15 & 18 & 7.5 \\
\hline Soya integral & 5 & 5 & 0 & 20 \\
\hline Semilla de linaza & 10 & 15 & 20 & 0 \\
\hline Carbonato de calcio & 10.2 & 10.22 & 10.15 & 10.41 \\
\hline Fosfato dicalcico & 1.01 & 0.97 & 1.03 & 0.7 \\
\hline Sal & 0.3 & 0.3 & 0.3 & 0.3 \\
\hline Cloruro de colina & 0.1 & 0.1 & 0.1 & 0.1 \\
\hline Premezcla de vitaminas y minerales & 0.1 & 0.1 & 0.1 & 0.1 \\
\hline Metionina & 0.25 & 0.24 & 0.22 & 0.29 \\
\hline \multicolumn{5}{|l|}{ Composición calculada } \\
\hline Energía metabolizable. kcal/kg & 2800 & 2800 & 2800 & 2800 \\
\hline Proteína. \% & 17 & 17 & 17 & 17 \\
\hline Extracto etéreo. $\%$ & 5.9 & 7.6 & 8.2 & 6.5 \\
\hline Fibra cruda. $\%$ & 3.4 & 3.7 & 3.8 & 3.4 \\
\hline Calcio. \% & 4.2 & 4.2 & 4.2 & 4.2 \\
\hline Fósforo disponible. \% & 0.375 & 0.375 & 0.375 & 0.375 \\
\hline Lisina digestible. \% & 0.82 & 0.815 & 0.815 & 0.823 \\
\hline Metionina digestible. $\%$ & 0.47 & 0.468 & 0.46 & 0.527 \\
\hline Metionina + cistina. $\%$ & 0.742 & 0.742 & 0.742 & 0.742 \\
\hline Treonina. \% & 0.574 & 0.6 & 0.59 & 0.621 \\
\hline Triptófano. \% & 0.18 & 0.19 & 0.19 & 0.2 \\
\hline
\end{tabular}

Determinación de ácidos grasos. Para determinar el perfil de ácidos grasos en la yema de huevo, se colectaron al azar 5 yemas de huevo de cada réplica, las cuales se mezclaron luego de manera homogénea. De esta mezcla se tomaron
$2 \mathrm{~g}$, los cuales se extrajeron con $40 \mathrm{ml}$ de cloroformo: metanol 2:1 (11). El extracto se filtró y se colectaron $20 \mathrm{ml}$ de filtrado a los cuales se agregaron $5 \mathrm{ml}$ de agua destilada. Esta mezcla se centrifugó a $3000 \mathrm{rpm}$ durante 20 
minutos. Se eliminó el sobrenadante acuoso y se tomó $1 \mathrm{ml}$ de la fase inferior orgánica en un tubo de ensayo previamente pesado, el cual se evaporó luego bajo una corriente suave de nitrógeno. La grasa extraída y seca se solubilizó con una solución de cloroformo: metanol 1:1 ( $1 \mathrm{ml}$ por cada $100 \mathrm{mg}$ de lípidos) y una alícuota de $50 \mu$ de esta solución de lípidos fue derivatizada con el reactivo de metil esterificación MethPrep II ${ }^{\circledR}$ (Alltech Associates Inc., Deerfield, IL, USA). Los metil-ésteres de ácidos grasos se analizaron en un cromatógrafo de gases Shimadzu GC-14A equipado con un detector de ionización de llama (260ㄷ).

La separación se llevó a cabo con una columna Supelco ${ }^{\circledR}$ Omegawax 320 , de $30 \mathrm{~m} \times 0.32 \mathrm{~mm} \times 0.25 \mu \mathrm{m}$ de grosor de película. La separación se realizó mediante una rampa de temperatura (temperatura inicial de $80^{\circ} \mathrm{C}, 10^{\circ} \mathrm{C} / \mathrm{min}$ hasta $190^{\circ} \mathrm{C}, 20 \mathrm{~min}$ a $190 \circ \mathrm{C}, 2 \circ \mathrm{C} / \mathrm{min}$ hasta $220^{\circ} \mathrm{C}$ y $\left.10 \mathrm{~min} 220^{\circ} \mathrm{C}\right)$. Se utilizó helio como gas transportador y la inyección se hizo en modo "split" (relación 1:50). Los metil-ésteres de los ácidos grasos se identificaron por comparación con los tiempos de retención de una mezcla estándar de ácidos grasos (Supelco 37 component FAME Mix, Inc., Bellefonte, PA, USA). Cada ácido graso se reportó como porcentaje del total de ácidos grasos identificados en cada muestra analizada.

Parámetros productivos. Se determinó el peso corporal al comienzo y fin del experimento y el consumo de alimento semanalmente. Se registró diariamente la producción de huevo y se estimó la ganancia de peso, el porcentaje de producción de huevo y la conversión de alimento ( $g$ de alimento consumido/ docena de huevo producido).

Evaluación sensorial del huevo. Se seleccionaron 25 degustadores que cumplieran con los requisitos para evaluar las características organolépticas del huevo, entre ellas, que no tuviera aversión por el huevo, que no fuera fumador y que no tuviera trastornos del tracto respiratorio. Se evaluó el color, el sabor y la textura del huevo.

Análisis estadístico. El estudio se realizó bajo un diseño al azar con 5 réplicas y 3 gallinas por réplica en cada tratamiento. Para la evaluación de la composición de ácidos grasos en la yema de huevo se seleccionaron 3 réplicas por tratamiento, cada réplica conformada por la mezcla de 5 yemas de huevo. Todos los parámetros se sometieron a análisis estadístico descriptivo. La evaluación sensorial se hizo mediante tablas de contingencia y prueba de $\mathrm{X}^{2}$ (12).

\section{RESULTADOS}

Composición de ácidos grasos saturados de la yema de huevo.Para todas las muestras evaluadas, el porcentaje de ácido palmítico (C16:0) fue relativamente alto cuando se compara con los otros ácidos grasos (Tabla 2). El grupo LO presentó el mayor contenido de ácido palmítico cuando se comparó con el grupo L20 $(p<0.01)$, con 24.6 y $22.1 \%$, respectivamente. El contenido de ácido esteárico (C18:0) no presentó diferencias en respuesta a los niveles de inclusión de semilla de lino $(p>0.05)$. El contenido de ácidos grasos saturados se redujo significativamente con la inclusión de semilla de lino $(p<0.05)$, debido a la reducción significativa de ácido palmítico.

Ácidos grasos monoinsaturados (MUFAs). El ácido oleico (C18:1) fue el más importante dentro del perfil en todos los tratamientos, siendo significativamente superior en el grupo experimental L10 con $43 \%(p<0.01)$. El ácido graso palmitoleico (C16:1), no presentó diferencias significativas entre los grupos experimentales $(p>0.05)$. El contenido total de MUFAs, estuvo entre un 42.3 y $46.7 \%$ del total de ácidos grasos identificados, sin presentar diferencias entre tratamientos $(p>0.05)$.

Ácidos grasos poliinsaturados $\mathbf{n - 6}$ (PUFAs n-6). El contenido de ácido linoleico (C18:2, n-6) se redujo significativamente en proporción al aumento del nivel de inclusión 
Tabla 2. Composición de ácidos grasos de la yema de huevo en aves de postura alimentadas con diferentes niveles de semilla de linaza en la dieta.

\begin{tabular}{|c|c|c|c|c|c|}
\hline \multirow[b]{2}{*}{ Acido graso } & \multicolumn{5}{|c|}{ Dieta experimental } \\
\hline & L10 & L15 & L20 & Lo & $P<a$ \\
\hline Mirístico. C14:0 & $0.2 \pm 0.1$ & $0.2 \pm 0.1$ & $0.2 \pm 0.1$ & $0.3 \pm 0.1$ & 0.67 \\
\hline Palmítito. C16:0 & $23.9 \pm 0.2 \mathrm{ab}$ & $23.3 \pm 0.1 b$ & $22.1 \pm 0.6 \mathrm{c}$ & $24.6 \pm 0.5 a$ & 0 \\
\hline Palmitoleico. C16:1 & $3.0 \pm 0.2$ & $3.3 \pm 0.2$ & $3.5 \pm 0.2$ & $2.9 \pm 0.1$ & 0.16 \\
\hline Esteárico. C18:0 & $7.7 \pm 0.3$ & $7.7 \pm 0.1$ & $8.0 \pm 0.6$ & $7.8 \pm 0.1$ & 0.89 \\
\hline Oleico. C18:1 n-9 & $43.4 \pm 0.5 a$ & $39.0 \pm 0.3 b$ & $38.5 \pm 0.1 b$ & $39.1 \pm 0.3 b$ & 0 \\
\hline Linoleico. C18:2 n-6 & $14.4 \pm 0.1 \mathrm{c}$ & $15.8 \pm 0.1 b$ & $14.4 \pm 0.3 c$ & $21.2 \pm 0.3 a$ & 0 \\
\hline Alfa-linolénico. C18:3 n-3 & $4.7 \pm 0.1 \mathrm{c}$ & $8.0 \pm 0.1 b$ & $10.3 \pm 0.9 a$ & $0.1 \pm 0.0 \mathrm{~d}$ & 0 \\
\hline Eicosapentaenoico. EPA. C20:5 n-3 & $0.1 \pm 0.0$ & $0.1 \pm 7.1$ & $0.1 \pm 0.0$ & ND & 0.39 \\
\hline Docosapentaenoico. DPA. C22:5 n-3 & $0.2 \pm 0.0 \mathrm{~b}$ & $0.2 \pm 0.0 \mathrm{ab}$ & $0.3 \pm 0.0 a$ & $0.1 \pm 0.0 \mathrm{c}$ & 0 \\
\hline Docosahexaenoico. DHA. C22:6 n-3 & $0.1 \pm 0.4$ & $1.3 \pm 0.1$ & $1.3 \pm 0.2$ & $0.7 \pm 0.0$ & 0.29 \\
\hline Docosadienoico. C22:2 & $0.1 \pm 0.0$ & $0.1 \pm 0.0$ & $0.1 \pm 0.0$ & $0.1 \pm 0.1$ & 0.79 \\
\hline Eicosenoico. C20:1 n-9 & $0.1 \pm 0.0 \mathrm{a}$ & $0.1 \pm 0.0 b$ & $0.1 \pm 0.0 \mathrm{c}$ & $0.1 \pm 0.0 \mathrm{a}$ & 0 \\
\hline Eicosadienoico. $C 20: 2$ & $0.1 \pm 0.0$ & $0.1 \pm 0.0$ & $0.1 \pm 0.0$ & $0.1 \pm 0.0$ & 0.18 \\
\hline Cis-11-14 icosatrienoico. C20:3 n-3 & $3.3 \pm 2.7$ & $0.5 \pm 0.2$ & $0.5 \pm 0.2$ & $1.2 \pm 0.0$ & 0.44 \\
\hline $\mathrm{SFA}^{3}$ & $31.7 \pm 0.2 \mathrm{ab}$ & $31.2 \pm 0.2 b$ & $30.4 \pm 0.6 b$ & $32.1 \pm 0.5 a$ & 0.01 \\
\hline MUFA $^{a}$ & $46.7 \pm 0.3$ & $38.9 \pm 3.7$ & $42.3 \pm 0.3$ & $42.4 \pm 0.3$ & 0.11 \\
\hline PUFA $^{7}$ & $21.6 \pm 0.1 \mathrm{c}$ & $26.3 \pm 0.2 a$ & $27.3 \pm 0.3 a$ & $24.6 \pm 0.2 b$ & 0 \\
\hline$n-6$ & $14.4 \pm 0.1 \mathrm{c}$ & $15.9 \pm 0.1 b$ & $14.5 \pm 0.3 \mathrm{c}$ & $21.3 \pm 0.3 a$ & 0 \\
\hline$n-3$ & $6.9 \pm 0.1 \mathrm{c}$ & $10.2 \pm 0.2 b$ & $12.6 \pm 0.6 a$ & $3.0 \pm 0.0 \mathrm{~d}$ & 0 \\
\hline$n 6: n 3$ & $2.1 \pm 0.05 b$ & $1.6 \pm 0.04 c$ & $1.2 \pm 0.08 \mathrm{~d}$ & $7.0 \pm 0.15 a$ & 0 \\
\hline
\end{tabular}

(a-d) diferentes letras en la fila indican diferencias significativas $(p<0.05) .{ }^{5}$ SFA: Ácidos grasos saturados. ${ }^{6}$ MUFA: Ácidos grasos monoinsaturados. ${ }^{7}$ PUFA: Ácidos grasos poliinsaturados.

de linaza en la dieta de las gallinas $(p<0.01)$ y fue el único representante de la serie de ácidos grasos n-6. El grupo L0 presentó un $21.2 \%$, en contraste con el grupo L20 que presentó un $14.4 \%$ de ácido linoleico en la yema del huevo.

Ácidos grasos poliinsaturados $\mathbf{n}-\mathbf{3}$ (PUFAs $\mathbf{n - 3 )}$ ). La respuesta más clara fue observada con el ácido $\alpha$-linolénico (C18:3n-3), cuyo contenido se incrementó de acuerdo con el aumento del nivel de inclusión de linaza en la dieta $(p<0.01)$. Así, el grupo LO se encontró una deposición de $0.1 \%$ de ácido $\alpha$-linolénico en la yema de huevo, mientras que el grupo L20 presentó un $10.3 \%$. El ácido graso EPA (C20:5, n-3) no fue detectado en el grupo LO. EI DPA (C22: $5, n-3)$ fue más bajo en el grupo LO $(p<0.01)$ y el DHA $(C 22: 6, n-3)$ se aumentó en un $86 \%$ en los grupos L15 y L20, respecto al grupo control LO. Igualmente, el grupo LO presentó un contenido total de ácidos grasos n-3 de $3.0 \%$, significativamente más bajo que los grupos L10, L15 y L20, Ios cuales presentaron un contenido total de ácidos grasos $\mathrm{n}-3$ de $6.9,10.2$ y $12.6 \%$, respectivamente.

Como consecuencia de la alteración de la composición de ácidos grasos n- 6 y n- 3 en la yema del huevo por efecto de la inclusión de linaza, la relación ácidos grasos n-6: $n-3$ se redujo de 7.0 para el grupo L0 hasta 1,2 para el grupo L20 $(p<0.01)$ (Tabla 2).

Producción de huevo. En la tabla 3 se observa que durante la semana 3 , los 
grupos L0 y L15 presentaron la mejor producción de huevos con 95.2 y $93.3 \%$, respectivamente, frente a los grupos L10 y L20, los cuales obtuvieron una producción de huevo de $85.7 \%$. Esta misma tendencia se observó durante la semana 6 y en la producción acumulada $(p<0.05)$.

Calidad del huevo. En la tabla 4 se observa que la inclusión de semilla de lino en la dieta de gallinas ponedoras, no afectó significativamente el peso promedio del huevo o el peso promedio de la yema. Sin embargo, el color de la yema se redujo significativamente, según la escala Roche; el grupo sin inclusión de semilla de lino presentó el mayor valor para pigmentación de yema de 8,5 con respecto a $4.0,4.25$ y 6.0 de los grupos L10, L15 y L20 $(p<0.05)$. No se encontraron diferencias significativas en la percepción de los degustadores en cuanto al sabor $(p>0.05)$

Tabla 3. Producción de huevo (\%) en aves de postura alimentadas con diferentes niveles de inclusión de semilla de linaza.

\begin{tabular}{cccccc}
\hline \multicolumn{5}{c}{ Dieta experimental } \\
\hline Semana & L10 & L15 & L20 & L0 & P<a \\
\hline 1 & $87.6 \pm 1.74^{*}$ & $90.5 \pm 2.46$ & $91.4 \pm 2.80$ & $92.4 \pm 3.69$ & 0.65 \\
2 & $91.4 \pm 3.47$ & $90.5 \pm 3.20$ & $92.4 \pm 2.26$ & $96.2 \pm 5.60$ & 0.74 \\
3 & $85.7 \pm 2.69 \mathrm{~b}$ & $93.3 \pm 3.25 \mathrm{ab}$ & $85.7 \pm 2.69 \mathrm{cb}$ & $95.2 \pm 1.90 \mathrm{a}$ & 0.03 \\
4 & $90.5 \pm 2.45$ & $93.3 \pm 2.90$ & $92.4 \pm 2.70$ & $94.3 \pm 4.46$ & 0.86 \\
5 & $81.0 \pm 3.06$ & $91.4 \pm 1.90$ & $81.9 \pm 6.30$ & $90.5 \pm 4.32$ & 0.19 \\
6 & $80.0 \pm 4.21 \mathrm{ac}$ & $90.0 \pm 4.7 \mathrm{a}$ & $72.2 \pm 4.68 \mathrm{bc}$ & $90.0 \pm 1.49 \mathrm{a}$ & 0.01 \\
Promedio acumulado & $86.0 \pm 1.94 \mathrm{~b}$ & $91.5 \pm 0.60 \mathrm{ab}$ & $86.0 \pm 3.25 \mathrm{~b}$ & $93.1 \pm 1.04 \mathrm{a}$ & 0.04 \\
\hline
\end{tabular}

* Promedio \pm error estándar, resultado de 5 replicas por tratamiento. (a-b) diferentes letras en la misma fila indican diferencias significativas $(p<0.05)$

Tabla 4. Características del huevo en aves de postura alimentadas con diferentes niveles de inclusión de semilla de linaza.

\begin{tabular}{|c|c|c|c|c|c|}
\hline \multirow[b]{2}{*}{ Parámetro } & \multicolumn{5}{|c|}{ Dieta experimental } \\
\hline & L10 & L15 & L20 & Lo & $P<a$ \\
\hline Peso del huevo. g & $66.2 \pm 0.56^{*}$ & $67.3 \pm 0.9$ & $63.4 \pm 0.88$ & $67.3 \pm 0.54$ & 0.53 \\
\hline Peso de la yema. g & $15.9 \pm 0.23$ & $15.6 \pm 0.16$ & $16.3 \pm 0.73$ & $16.3 \pm 0.10$ & 0.52 \\
\hline Peso cáscara. g & $6.7 \pm 0.20$ & $6.8 \pm 0.15$ & $6.7 \pm 0.06$ & $7.1 \pm 0.30$ & 0.52 \\
\hline $\begin{array}{l}\text { Color de la yema } \\
\text { (Escala de Roche) }\end{array}$ & $4.0 \pm 1.08 b$ & $\begin{array}{c}4.25 \pm \\
0.25 b\end{array}$ & $6.0 \pm 0.41 b$ & $8.5 \pm 0.5 a$ & 0 \\
\hline
\end{tabular}

* Promedio \pm error estándar, resultado de 5 réplicas por tratamiento. (a-b) diferentes letras indicando en la fila las diferencias significativas de los tratamientos $(p<0.05)$.

\section{SCUSIÓN}

El efecto relevante de la inclusión de semilla de linaza sobre la composición de ácidos grasos en la yema de huevo fue una reducción significativa de ácido linoleico acompañada de un aumento significativo de ácidos grasos n-3, principalmente el acido $\alpha$-linolénico. Similares resultados reportaron
Leeson et al (13). Sin embargo, los resultados del presente estudio mostraron niveles de enriquecimiento superiores a los reportados por Cherian y $\operatorname{Sim}$ (14) quienes encontraron $5.77 \%$ de ácido $\alpha$-linolénico en la grasa de la yema de huevo de gallinas suplementadas con $10 \%$ de semilla de lino. Contrariamente, Aymond y Van Elswyk (15) concluyeron que la deposición de ácido $\alpha$ - 
linolénico en el huevo no fue afectada por la inclusión de linaza hasta un $10 \%$. Por otra parte, Galobart et al (16) obtuvieron un $11.85 \%$ de ácido $\alpha$-linolénico con $5 \%$ de inclusión de aceite de lino. Se logró una relación 47: 1 de ácido $\alpha$-linolénico en yema del huevo del grupo alimentado con $15 \%$ de semilla de lino respecto al grupo control. En el presente estudio, con la inclusión de linaza en la dieta de las aves, fue posible reducir en 5.8 veces la relación entre ácidos grasos n-6 y n-3, aspecto favorable desde la perspectiva de la salud humana.

La inclusión de semilla de lino en la dieta de las gallinas permitió incrementar el contenido de PUFAs en la yema de huevo de manera similar a la reportada por Ayerza et al (17). Los mismos autores (18) establecieron además que un alto consumo dietario de PUFAs puede causar una disminución en la síntesis de ácidos grasos saturados. I gualmente, Cherian et al (19) concluyeron que el contenido de ácidos grasos saturados es inversamente proporcional al contenido de PUFAs en la dieta. Similares resultados se observaron en el presente estudio, es decir que a medida que se incrementó el nivel de inclusión de semilla de lino, el contenido de PUFAs en la yema de huevo se redujo. Por el contrario, Ayerza y Coates (18), reportaron que el total de ácidos grasos saturados, calculado como la suma de los ácidos mirístico, palmítico y esteárico, no fue significativamente diferente entre grupos que consumieron dietas con diferentes niveles de inclusión de semilla de lino. Esta discrepancia estaría explicada porque en el presente estudio, las dietas formuladas con semilla de lino fueron con base en sorgo como fuente de energía, a diferencia de la dieta control que fue formulada con maíz. El sorgo es muy bajo en grasa, en cambio el maíz tiene un 5.6\% de grasa, la cual tiene alto contenido de ácido palmítico (20). En general, en todos los grupos experimentales, el contenido de ácidos grasos saturados fue relativamente alto, osciló entre 30.4 y $32.1 \%$.

El contenido de MUFAs no fue consistente con los niveles de inclusión de semilla de lino en la dieta de las gallinas, en contraste, Ayerza y Coates (18) sí encontraron una reducción de estos ácidos grasos en yema de huevo de las gallinas alimentadas con dietas enriquecidas con ácido $\alpha$-linolénico.

En el presente estudio, con un $15 \%$ de inclusión de linaza, no se afectó el porcentaje de producción de huevo. Similares resultados reportaron Caston et al (6), en un estudio más prolongado (48 semanas) en el cual no se observó disminución en la producción de huevos cuando evaluaron una inclusión de 10 ó 20\% de linaza molida. Galobart et al (16) observaron que la tasa de postura de las gallinas se situó alrededor del $90 \%$ cuando consumieron una dieta con $4 \%$ de inclusión de aceite de linaza; similares resultados reportaron Yannakopoulos et al (19), quienes observaron un $90 \%$ de producción de huevos por más de 8 semanas. En el presente estudio se obtuvo un porcentaje de producción de $91.5 \%$ con $15 \%$ de inclusión de linaza. Por el contrario, Scheideler y Froning (7), encontraron que gallinas ponedoras alimentadas con 5 y $15 \%$ de linaza presentaron una producción de huevos del $88.9 \%$ comparada con gallinas que se alimentaban con dietas tradicionales, las cuales tuvieron una producción de huevos de $83.1 \%$.

Por otra parte, Leeson (1) y Yannakopoulos et al (20) reportaron que dietas con 10 ó $20 \%$ de inclusión de linaza, no causan ningún efecto sobre la producción de huevo o el peso del huevo en gallinas ponedoras. Estos resultados tan contrastantes estarían explicados por varios de los múltiples factores que están asociados con la producción de huevos, entre ellos, la calidad del levante, la concentración de nutrientes en la dieta, la temperatura, el alojamiento, entre otros (21).

La conversión de alimento estuvo asociada con el porcentaje de producción de huevos y se observó una mejor conversión en los grupos L15 y L0 frente a los dos grupos experimentales. Contrariamente a lo observado en el presente estudio, Schumann et al (22), encontraron que las gallinas alimentadas con $10 \%$ de linaza y $4 \%$ de aceite de linaza presentaron una mejor conversión de alimento con respecto al grupo 
control. Este efecto podría estar explicado por el efecto extra calórico de la dieta asociado con un mayor contenido de grasa aportada por la semilla de lino (12). A pesar de que se reporta un efecto laxante de la semilla de linaza, debido a que contiene un mucílago (fibra soluble), en el presente estudio no se observó presencia de diarreas en las gallinas.

En cuanto a las características organolépticas del huevo, la inclusión de semilla de lino no afectó negativamente el sabor, resultado que concuerda con los de Caston y Leeson (23) quienes reportan que es improbable que la incorporación de linaza a las dietas de las gallinas conduzca a la producción huevos con características organolépticas indeseables. Sin embargo, otros autores reportaron efectos indeseables sobre el sabor del huevo causados por la suplementación de semilla de lino, por ejemplo, Cruickshank (24), trabajando con una inclusión de $28 \%$ de aceite de lino y Jiang et al (25) con $15 \%$ de inclusión de linaza en la dieta, demostraron la presencia de sabores desagradables en los huevos.

Todos los grupos con inclusión de semilla de lino presentaron un color de la yema en la escala de color Roche por debajo del valor aceptable para huevos de grado $A$ en la mayoría de países (7-8,21). Este resultado sería posiblemente explicado porque las dietas para los grupos L10, L15 y L20, se hicieron con sorgo como fuente de energía, mientras que la dieta control se formuló con maíz, el cual tiene mayor aporte de pigmentantes.

En conclusión, la producción de huevo enriquecido con ácidos grasos omega- 3 es viable, con un sobrecosto de producción de $12 \%$. Con un $15 \%$ de inclusión de linaza en la dieta de gallinas ponedoras se incrementa el contenido total de ácidos grasos omega3 y se aumenta 80 veces el contenido de ácido $\alpha$-linolénico, sin afectar negativamente el sabor, el peso y la textura del huevo. A este nivel de inclusión, sin embargo, se reduce la pigmentación del huevo, por lo que es necesario incrementar el contenido de pigmentantes y antioxidantes para conservar el color en la escala óptima.

\section{Agradecimientos}

A Carolina Tello y Diego Guerrero y al Centro de Investigación y Capacitación San Miguel de La Universidad de La Salle, Bogatá, Colombia.

\section{REFERENCI AS}

1. Leeson S. Potential of modifying poultry products. JAPR 1993; 2: 380-385.

2. Walzem RL. Lipoproteins and the laying hen: form follows function. Poult Avian Biol Rev 1996; 7:31 -64.

3. Baucells MD, Crespo N, Barroeta AC, López-Ferrer S, Grashorn MA. Incorporation of Different Polyunsaturated Fatty Acids into Eggs. Poult Sci 2000; 78: 51-59.

4. Noble RC, Cochi M. Lipid metabolism and the neonatal chicken. Prog Lipid Res 1990; 29: 107-140.
5. Patterson P, Koelkebeck D, Bell J, Carey K, Darre M. Egg marketing national supermarkets: specialty eggs- Part 2. Poult Sci 2001; 80: 390-395.

6. Caston LJ, Squires EJ and Leeson S. Hen performance, egg quality and the sensory evaluation of eggs from SCWL hens fed dietary flax. Can J of Anim Sci 1994; 74:347-353.

7. Scheideler SE, Froning GW. The combined influence of dietary flaxseed variety, level, form, and storage conditions on egg production and composition among vitamin E-supplemented hens. Poult Sci 1996; 75: 1221-1226. 
8. Scheideler SE, Froning G, Cuppett S. Effect of dietary flaxseed and fish oil on egg components, sensory analysis and oxidation products. Poult Sci 1994; Suppl 1, 73: 118.

9. Diaz GJ. Naturally occurring toxins relevant to poultry nutrition. In: Leeson, S., J.D. Summers, editores. Scott's Nutrition of the Chicken, 4 ed. Guelph: University Books; 2001. p. 544-591.

10. Rostagno HS, Albino LF, Donzele JL. Composição de alimentos e exigências nutricionais. Tabelas brasileiras para aves e suínos. Viçosa: Editora UFV; 2005.

11. Folch J, Lees M, Sloane G. A simple method for the isolation and purification of total lipids from animal tissues. J Biol Chem 1957; 226: 497-507.

12. Snedecor GW and Cochran W G. Statistical Methods. 7 ed. I owa: Iowa State University Press; 1980.

13. Leeson S, Summers J. Nutrition of the Chicken. 4ed. Ontario: University Books; 2001.

14. Cherian G, Sim J. Effect of feeding full fat flax and canola seeds to laying hens on the fatty acid composition of eggs, ambryos and wewly hatched chicks. Poult Sci 2000; 70: 917-922.

15. Aymond WM, Van Elswyk ME. Yolk thiobarbituric acid reactive substances and $\mathrm{n}-3$ fatty acids in response to whole and ground. Poult Sci 1995; 80: 14961505.

16. Galobart J, Barroeta AC, Baucells MD, Cortinas L, Guardiola F. á-tocopherol transfer efficiency and lipid oxidation in fresh and spray-dried eggs enriched with ù3-polyunsaturated fatty acids. Poult Sci 2001; 80: 1496-1505.
17. Ayerza R, Coates W, Laura M. Semilla Chia (Salvia Hispánica) como fuente de ácidos grasos $\mathrm{n}-3$; contenido de colesterol en la carne de pollo, Poult Sci 1999; 79:53-58.

18. Ayerza R, Coates W. Omega-3 enriched eggs: The influence of dietary álinolenic fatty acid source on egg production and composition. Can J Anim Sci 2001; 81:355-361.

19. Cherian G, Li SX, Sim JS. Dietary alphalinolenic acid and laying hen strain: Fatty acids of liver, adipose tissue, white meat, dark meat and egg yolk. J Agric Food Chem 1995; 43: 2553-2559.

20. Yannakopoulos A, Tserveni-Gousi A, Christaki E. Enhanced egg production in practice: the case of bio-omega-3 egg. Int J of Poult Sci 2005; 4:531-535.

21. Leeson S, Summers J, Díaz GJ . Nutrición aviar comercial. Bogotá (D.C); Le'print Club Express Ltda; 2000.

22. Schumann BE, Squires EJ, Leeson S. Effect of dietary flaxseed, flax oil and n3 fatty acid supplement on hepatic and plasma characteristics relevant to fatty liver haemorrhagic syndrome in laying hens. Br Poult Sci 2000; 41: 465-473.

23. Caston L, Leeson S. Dietary flaxseed and egg composition. Poult Sci 1990; 69: 1617-1620.

24. Cruickshank EM. Studies in fat metabolism in the fowl: the composition of the egg fat and depot fat of the fowl as affected by the ingestion of large amounts of different fats. Biochem J 1934; 28: 965-77.

25. Jiang Z, Ahn DU, Sim J S. Effects of feeding flax and two types of sunflower seeds on fatty acid compositions of yolk lipid classes. Poult Sci 1991; 70: 2467-2475. 